\title{
Anisotropic Dark Matter Stars
}

\author{
P.H.R.S. Moraes, ${ }^{1, \text { f }}$ G. Panotopoulos, ${ }^{2}$, and I. Lopes ${ }^{2}$, 用 \\ ${ }^{1}$ Universidade de São Paulo (USP), Instituto de Astronomia, \\ Geofísica e Ciências Atmosféricas (IAG), Rua do Matão 1226, \\ Cidade Universitária, 05508-090 São Paulo, SP, Brazil \\ ${ }^{2}$ Centro de Astrofísica e Gravitação-CENTRA, Departamento de Física, Instituto Superior Técnico-IST, \\ Universidade de Lisboa-UL, Av. Rovisco Pais, 1049-001 Lisboa, Portugal
}

\begin{abstract}
The properties of exotic stars are investigated. In particular, we study objects made entirely of dark matter and we take into account intrinsic anisotropies which have been ignored so far. We obtain exact analytical solutions to the structure equations and we we show that those solutions i) are well behaved within General Relativity and ii) are capable of describing realistic astrophysical configurations.
\end{abstract}

PACS numbers:

\section{INTRODUCTION}

Dark matter (DM) has certainly been one of the greatest mysteries of Physics. An important evidence of its existence came from the analysis of rotation curves of spiral galaxies by V. Rubin and collaborators in the 70's of the last century 1 3. DM is thought to be a kind of matter that does not interact electromagnetically and therefore cannot be seen, which is why it is called dark. However, it interacts gravitationally. In the case of spiral galaxies, it causes their rotation curves to be significantly higher than one would expect by measuring only the gravitational field of luminous matter.

It also has a fundamental role in the formation of galaxies and large-scale structures in the universe [4 7]. Actually it is believed that when baryonic matter decoupled from radiation at redshift $z \sim 1100$, the DM gravitational potential wells were already formed and rapidly attracted baryonic matter, what has speeded up the structure formation mechanism [8, 9] so that we can see the large-scale structures we see today.

Moreover, according to standard cosmological model matched with observational data coming from temperature fluctuations in the cosmic microwave background radiation, DM makes up roughly $25 \%$ of the matter density of entire universe composition [10].

We still have not detected DM particles with experimental apparatus despite the efforts $11-14$. So far we have only detected its gravitational effects when pointing telescopes to the sky. On this latter regard, gravitational lensing has been fundamental [15 17.

At least a portion of DM may be in the form of massive compact halo objects or MACHOs [18 20]. Those are massive baryonic matter objects that emit low or no electromagnetic radiation and habit galactic halos,

\footnotetext{
*Electronic address: moraes.phrs@gmail.com

${ }^{\dagger}$ Electronic address: grigorios.panotopoulos@tecnico.ulisboa.pt

$\ddagger$ Electronic address: ilidio.lopes@tecnico.ulisboa.pt
}

and an example of them would be neutron stars. They can also bend light, causing gravitational microlensing effects, that have been detected for some time [21 25].

It should also be quoted that DM gravitational effects could be understood as purely geometrical effects of extended gravity theories [26]. Rotation curves [27- 29] and even structure formation 30,34 have been explained through the extended gravity channel.

Here, in the present article, based on some of the several studies that empirically prove DM existence [35, we are going to stick to the standard approach, considering DM exists and is non-baryonic.

The Bose-Einstein condensate is a possibility in the DM particle scenario 36 40, and it was recently shown that could exist in space by the Cold Atom Laboratory orbiting Earth on board the International Space Station 41.

The weakly interacting massive particles (so-called WIMPs) 42 44 are among the best motivated DM particle candidates. WIMPS interact through a feeble new force and gravity as predicted by supersymmetry among other theories [45, 46]. If they were in thermal equilibrium in the early universe they annihilated with one another so that a predictable number of them remains today 47.

There may exist DM stars (DMSs) 48, 49] powered by WIMP DM annihilation [50, 51. In regions of high DM density, such as the Galactic center, the capture and annihilation of WIMP DM by stars has the potential to significantly alter their evolution 5255 . In Reference [56. it was shown that WIMPs accreted onto neutron stars may provide a mechanism to seed strangelets in compact objects for WIMP masses above a few GeV. This effect may trigger a conversion of most of the star into a strange star. Recall that neutron stars are pulsars, highdensity stars with large rotation frequency rates located in the core of supernovae remnants [57, 58]. Some models predicted that strange stars could form inside these stars due to the brake neutrons into their constituent quarks [59]. Due to a matter of stability, a portion $(\sim 1 / 3)$ 
of these quarks is converted to strange quarks and the resulting matter is known as strange quark matter.

Neutron stars are expected to efficiently capture WIMPs due to their strong gravitational field. The annihilation of DM in the center of these stars could lead to detectable effects on their surface temperature, specially if they are in the center of our Galaxy 60].

In 61, Kurita and Nakano investigated the collapse of clusters of WIMPs in the core of Sun-like stars and the consequent possible formation of mini-black holes, which would generate gravitational wave emission.

The aforementioned Bose-Einstein condensate has also been considered as the DM modeling for stars. On this regard, one can consult e.g. 62 69. In particular, in 68, 69, DMSs were investigated in the Starobinsky model of gravity [70. It has been shown in 69] that DMSs have smaller radius and are slightly more massive in Starobinsky gravity.

In the present article we will assume a boson star as our model for DMS. A wide variety of boson stars have been proposed and investigated in the literature $71+75$ (for some recent references on this subject, one can check [76-79]). Our DMS will be modeled from the equation of state (EoS) proposed in [75] (check also 80]). It is interesting to mention that some proposals for detecting boson stars were reported in 81 86.

The environment inside DMSs is expected to be extremely dense, specially when neutron star-like objects are under consideration. Under such conditions of extreme density, anisotropy is expected to appear [87 90].

Anisotropy in neutron stars has been investigated in the literature. The hydrostatic features were firstly approached in 91, where it was shown that deviations from isotropy would entail changes in the star maximum mass. This approach was extended in [92] to also cover the problem of stability under radial and non-radial pulsations. The effects of anisotropy on slowly rotating neutron stars was studied in 93. In 94, anisotropic neutron stars were also considered in the framework of Starobinsky gravity. Further studies of anisotropic neutron stars can be seen in References 95 98 .

To the best knowledge of the present authors, anisotropy has not yet been considered in DMSs. Such an investigation is the main goal of the present article. The plan of our work is the following: in the next section we briefly summarize the structure equations describing hydrostatic equilibrium of anisotropic stars. In Section III we present the exact analytical solution and we show that it is well behaved and realistic within General Relativity. Finally, we finish our work in Section 4 with the concluding remarks.

\section{RELATIVISTIC STARS WITH ANISOTROPIC MATTER}

Within General Relativity the starting point is Einstein's field equations

$$
\mathcal{G}_{\mu \nu}=\mathcal{R}_{\mu \nu}-\frac{1}{2} \mathcal{R} g_{\mu \nu}=8 \pi T_{\mu \nu}
$$

In (1), $\mathcal{G}_{\mu \nu}$ is the Einstein tensor, $\mathcal{R}_{\mu \nu}$ is the Ricci tensor, $\mathcal{R}$ is the Ricci scalar, $g_{\mu \nu}$ is the metric tensor, we set Newton's constant $G$ and the speed of light, $c$, to 1, while for anisotropic matter the stress-energy tensor, $T_{\mu \nu}$, has the form

$$
T_{\nu}^{\mu}=\operatorname{Diag}\left(-\rho, p_{r}, p_{t}, p_{t}\right),
$$

with $\rho$ being the energy density, $p_{r}$ the radial pressure and $p_{t}$ the tangential pressure.

In order to find interior solutions describing hydrostatic equilibrium of relativistic stars, we integrate the structure equations including the presence of a nonvanishing anisotropic factor [99, 100]:

$$
\begin{aligned}
m^{\prime}(r) & =4 \pi r^{2} \rho(r), \\
\nu^{\prime}(r) & =2 \frac{m(r)+4 \pi r^{3} p_{r}(r)}{r^{2}[1-2 m(r) / r]} \\
p_{r}^{\prime}(r) & =-\left[\rho(r)+p_{r}(r)\right] \frac{m(r)+4 \pi r^{3} p(r)}{r^{2}[1-2 m(r) / r]}+\frac{2 \Delta}{r},
\end{aligned}
$$

where $m(r)$ and $\nu(r)$ are the components of the metric tensor assuming static, spherically symmetric solutions in Schwarzschild-like coordinates, $(t, r, \theta, \phi)$,

$$
d s^{2}=-e^{\nu} d t^{2}+\frac{1}{1-2 m(r) / r} d r^{2}+r^{2}\left(d \theta^{2}+\sin ^{2} \theta \mathrm{d} \phi^{2}\right),
$$

and $\Delta \equiv p_{t}-p_{r}$ is the anisotropic factor. All quantities depend on the radial coordinate $r$ only, and a prime denotes differentiation with respect to $r$. Clearly, setting $\Delta=0$ we recover the usual Tolman-Oppenheimer-Volkoff equations [101, 102] for isotropic matter.

Moreover we impose at the center of the star, $r=0$, the following initial conditions

$$
\begin{aligned}
m(0) & =0, \\
p(0) & =p_{c},
\end{aligned}
$$

with $p_{c}$ being the central pressure. Upon matching with the exterior vacuum solution $\left(T_{\mu \nu}=0\right.$, Schwarzschild geometry) at the surface of the star, $r=R$, the following boundary conditions must be satisfied

$$
\begin{aligned}
p(R) & =0, \\
m(R) & =M, \\
e^{\nu(R)} & =1-\frac{2 M}{R},
\end{aligned}
$$

with $R$ being the radius of the star, and $M$ being its mass. 


\section{ANISOTROPIC DARK MATTER STARS: EXACT ANALYTICAL SOLUTION}

Boson stars are self-gravitating clumps made of either spin-zero fields, called scalar boson stars 72 or vector bosons, called Proca stars [103, 104. The maximum mass for scalar boson stars in non-interacting systems was found in [105, 106], while in [75, 107] it was pointed out that self-interactions can cause significant changes.

A complex scalar field, $\Phi$, minimally coupled to gravity is described by the Einstein-Klein-Gordon action [108]

$$
\begin{aligned}
S & =\int d^{4} x \sqrt{-g}\left(\frac{R}{16 \pi}+\mathcal{L}_{M}\right) \\
\mathcal{L}_{M} & =-g^{\mu \nu} \partial_{\mu} \Phi \partial_{\nu} \Phi^{*}-V(|\Phi|)
\end{aligned}
$$

where $g$ is the metric determinant, $\mathcal{L}_{M}$ is the matter lagrangian and $V$ is the self-interaction scalar potential.

For static spherically symmetric solutions we make for the scalar field the ansatz 108

$$
\Phi(r, t)=\phi(r) \exp (-i \omega t)
$$

where the oscillation frequency $\omega$ is a real parameter.

Although the scalar field itself depends on time, its stress-energy tensor is time independent and the Einstein's field equations take the usual form for a fluid, for which the energy density is computed to be [109, 110.

$$
\rho=\omega^{2} e^{-\nu} \phi^{2}+e^{-\lambda} \phi^{2}+V(\phi),
$$

while the radial and tangential pressures are found to be 109, 110 .

$$
\begin{aligned}
& p_{r}=\omega^{2} e^{-\nu} \phi^{2}+e^{-\lambda} \phi^{2}-V(\phi), \\
& p_{t}=\omega^{2} e^{-\nu} \phi^{2}-e^{-\lambda} \phi^{\prime 2}-V(\phi) .
\end{aligned}
$$

Clearly, a boson star is anisotropic since the two pressures are different. Under certain conditions, however, the anisotropy may be ignored and the system can be treated as an isotropic object. A concrete model of the form

$$
V(|\Phi|)=m^{2}|\Phi|^{2}+\frac{\lambda}{2}|\Phi|^{4},
$$

with $m$ being the mass of the scalar field and $\lambda$ being the self-interaction coupling constant, was studied e.g. in 80, in which the authors considered the following EoS 75]:

$$
p_{r}=\frac{\rho_{0}}{3}\left(\sqrt{1+\frac{\rho}{\rho_{0}}}-1\right)^{2}
$$

where $\rho_{0}$ is a constant given by

$$
\rho_{0}=\frac{m^{4}}{3 \lambda} .
$$

This EoS describes the boson stars that are approximately isotropic provided that the condition

$$
\frac{\lambda}{4 \pi} \gg m^{2}
$$

holds 80].

In the two extreme limits we recover the well-known results

$$
p_{r} \approx \frac{\rho^{2}}{12 \rho_{0}}, \quad \rho \ll \rho_{0},
$$

for diluted stars 39, and

$$
p_{r} \approx \frac{\rho}{3}, \quad \rho \gg \rho_{0}
$$

in the ultra relativistic limit.

In the first extreme limit, any model, irrespectively of the form of the potential, will be described by the same polytropic EoS, with index $n=1$ and $\gamma=2$. In the present work we propose to investigate the properties of relativistic stars made of anisotropic exotic matter characterized by the polytropic EoS

$$
p_{r}=K \rho^{2}, \quad K=z / B
$$

where $z$ is a dimensionless number while $B$ has dimension of pressure and it is of the order of the energy density of neutron stars and quark stars, $B \simeq(150 \mathrm{MeV})^{4}$.

In the case of stars with anisotropic matter there are five unknown quantities in total and only three differential equations. Therefore, we are free to impose two conditions. Given the EoS, the simplest thing to do is to assume a certain profile for the energy density. In the following we shall consider the ansatz

$$
\rho(r)=\rho_{c}\left(1-\frac{r^{2}}{R^{2}}\right),
$$

which ensures that the energy density starts from a finite value at the origin, which is the central value $\rho_{c}$, and it monotonically decreases with $r$, until it vanishes at the surface of the star.

Now, all the other quantities may be computed one by one using the structure equations and the EoS. In particular, the radial pressure is immediately computed making use of the EoS, while the mass function is computed using the $t t$ component of the field equations, and it is given by

$$
m(r)=4 \pi \int_{0}^{r} d x x^{2} \rho(x)=4 \pi \rho_{c} r^{3}\left(\frac{1}{3}-\frac{r^{2}}{5 R^{2}}\right) .
$$

The temporal metric component $\nu$ is computed making use of the radial field equation as follows

$$
\nu(r)=\log (1-2 M / R)-2 \int_{R}^{r} d x \frac{m(x)+4 \pi x^{3} p_{r}(x)}{x^{2}[1-2 m(x) / x]} .
$$

Finally, the anisotropic factor is computed making use of the conservation of energy

$$
\left.\Delta(r)=\frac{r}{2}\left\{\left[p_{r}(r)+\rho(r)\right] \frac{m(r)+4 \pi r^{3} p_{r}(r)}{r^{2}[1-2 m(r) / r)}+p_{r}^{\prime}(r)\right]\right\},
$$

while the tangential pressure is computed from $p_{t}(r)=$ $p_{r}(r)+\Delta(r)$.

Next we shall investigate the behavior as well as the viability of the solutions we just found. 


\section{A. Causality, stability and energy conditions}

The radial and tangential speeds of sound, defined by

$$
\begin{aligned}
c_{r}^{2} & \equiv \frac{d p_{r}}{d \rho}, \\
c_{t}^{2} & \equiv \frac{d p_{t}}{d \rho}
\end{aligned}
$$

should take values in the interval $0<c_{r, t}^{2}<1$ throughout the stars, so that causality is not violated.

Moreover, Bondi suggested that for a stable Newtonian sphere the radial adiabatic index, defined by

$$
\Gamma \equiv c_{r}^{2}\left(1+\frac{\rho}{p_{r}}\right),
$$

should be larger than $4 / 3$ [11].

Finally, the solutions obtained here should be able to describe realistic astrophysical configurations. Therefore, as a further check we investigate if the energy conditions are fulfilled or not. To that end, the conditions [112 116

$$
\begin{gathered}
\rho \geq 0, \\
\rho+p_{r, t} \geq 0, \\
\rho-p_{r, t} \geq 0, \\
E_{+} \equiv \rho+p_{r}+2 p_{t} \geq 0, \\
E_{-} \equiv \rho-p_{r}-2 p_{t} \geq 0,
\end{gathered}
$$

are investigated.

Our main numerical results are summarized in Figures 1., 2 and 3 below, assuming the following numerical values for $z, B$ and $\rho_{c}$ :

$$
\begin{aligned}
B & =2 \times 10^{-80} m_{p l}^{4}, \\
\rho_{c} & =14 B \\
z & =0.009
\end{aligned}
$$

with $m_{p l}$ being the Planck mass. (27) and (28) correspond to a star with the following properties

$$
\begin{aligned}
R & =12 \mathrm{~km} \\
M & =2.08 M_{\odot}, \\
C & =0.258
\end{aligned}
$$

where $C=M / R$ is the compactness factor of the star.

In particular, Fig. 1 shows the normalized anisotropic factor $\Delta(r) / B$ versus $r / R$. It vanishes both at the center and at the surface of the star and it is positive throughout the object. The adiabatic index $\Gamma$ versus $r / R$ is shown in Fig. 2, where the Newtonian limit of $4 / 3$ is shown as well.

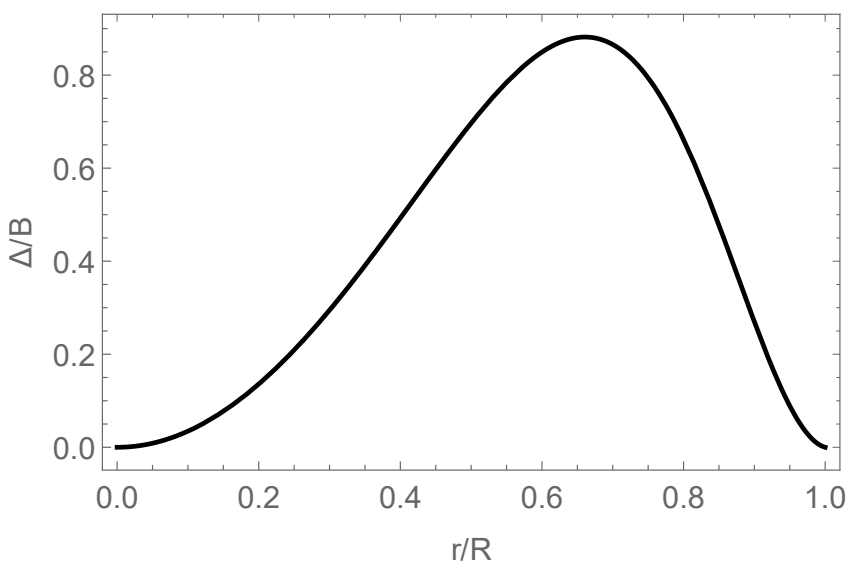

FIG. 1: Normalized anisotropic factor, $\Delta / B$, versus normalized radial coordinate $r / R$.

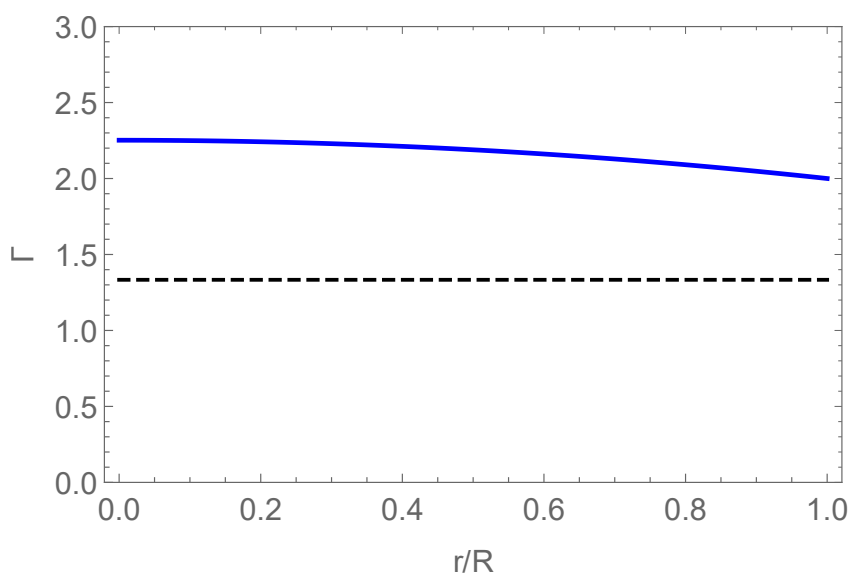

FIG. 2: Relativistic adiabatic index, $\Gamma$, versus normalized radial coordinate $r / R$. The horizontal line corresponds to the Newtonian limit of $4 / 3$.

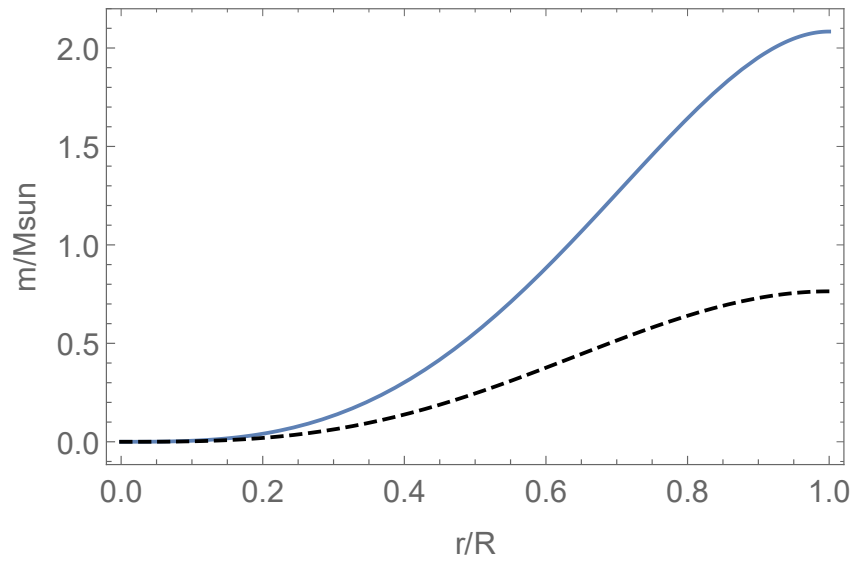

FIG. 3: Mass function (in solar masses) versus normalized radial coordinate $r / R$. The dashed curve corresponds to isotropic stars. 


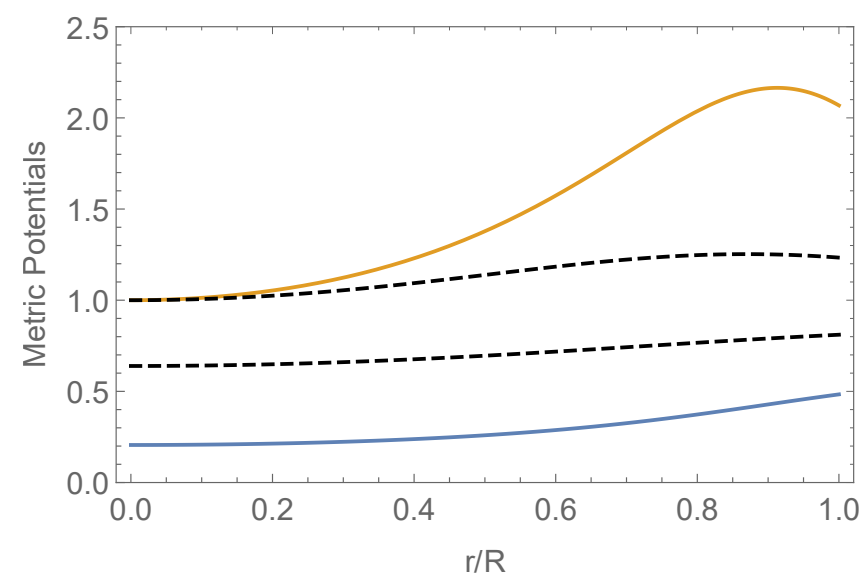

FIG. 4: The two metric components, $e^{\nu}$ (lower curve) and $1 /(1-2 m / r)$ (upper curve) versus normalized radial coordinate $r / R$. The dashed curves correspond to isotropic stars.

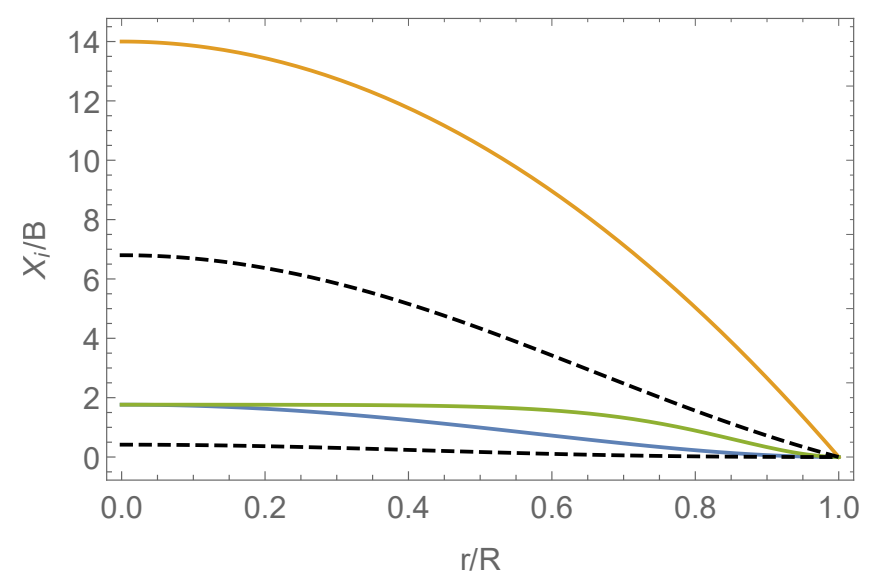

FIG. 5: Energy density $\rho / B$ (orange curve) radial pressure $p_{r} / B$ (blue curve) and tangential pressure $p_{t} / B$ (green curve) versus $r / R$. The dashed curves correspond to isotropic stars, where energy density lies above pressure.

In the Figures 3-6 a comparison is made between stars with anisotropic matter and their isotropic counterparts with the same EoS and the same radius. In particular, in Fig. 3 we show the mass functions versus $r / R$, while Fig. 4 shows the two metric potentials versus $r / R$. Finally, Fig. 5 shows normalized energy density and pressures versus $r / R$, while in Fig. 6 we show the speeds of sound, both radial (blue curve) and tangential (orange curve), versus $r / R$.

Clearly, causality is not violated as both sound speeds take values in the range $(0,1)$ throughout the star. More- over, the condition $\Gamma>4 / 3$ is satisfied as well. Finally, since both pressures are positive and lower than the energy density, all energy conditions are fulfilled.

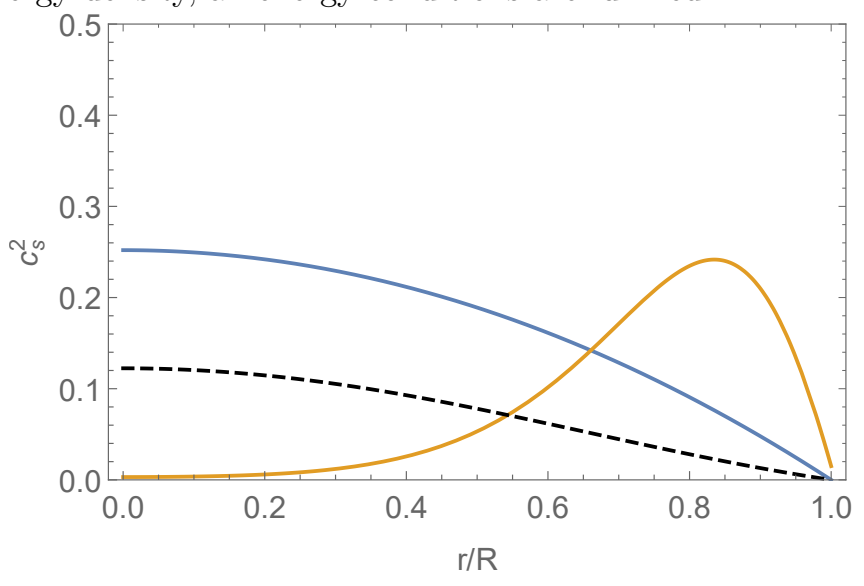

FIG. 6: Radial (blue curve) and tangential (orange curve) sound speeds, $c_{r}^{2}, c_{t}^{2}$, versus normalized radial coordinate $r / R$. The dashed curve corresponds to isotropic stars.

\section{CONCLUSIONS}

In summary, in the present work we have studied exotic stars with anisotropic matter within General Relativity. We have investigated in detail the properties of dark matter-type configurations, taking into account the presence of anisotropies. Exact analytic expressions for all the quantities of interest, such as mass function, anisotropic factor, relativistic index, speed of sound etc, have been found. Causality, stability criteria and energy conditions are also discussed. It is found that the solutions obtained here are well-behaved solutions capable of describing realistic astrophysical configurations. Finally, a direct comparison with their isotropic counterparts was made as well.

\section{Acknowledgments}

PHRSM thanks CAPES for financial support. The authors G. Panotopoulos and I. Lopes thank the Fundação para a Ciência e Tecnologia (FCT), Portugal, for the financial support to the Center for Astrophysics and Gravitation-CENTRA, Instituto Superior Técnico, Universidade de Lisboa, through the Project No. UIDB/00099/2020 and grant No. PTDC/FIS$\mathrm{AST} / 28920 / 2017$.
[1] V.C. Rubin et al., Astrophys. J. 225, L107 (1978).

[2] V.C. Rubin et al., Astrophys. J. 230, 35 (1979).

[3] C.J. Peterson et al., Astrophys. J. 226, 770 (1978).
[4] W. Hu, Astrophys. J. 506, 485 (1998).

[5] G.R. Blumenthal et al., Nature 311, 517 (1984).

[6] C.S. Frenk et al., Astrophys. J. 327, 507 (1988). 
[7] J.M. Gelb and E. Bertschinger, Astrophys. J. 436, 467 (1994).

[8] B. Ryden, Introduction to Cosmology (Addison Wesley, San Francisco, USA, 2003).

[9] S. Dodelson, Modern Cosmology (Academic Press, Amsterdam, Netherlands, 2003).

[10] Planck Collaboration, Astron. Astrophys. 594, A13 (2016).

[11] J. Aalbers et al., J. Cosm. Astrop. Phys. 11, 17 (2016).

[12] R. Harnik et al., J. Cosm. Astrop. Phys. 7, 26 (2012).

[13] F. Ruppin et al., Phys. Rev. D 90, 083510 (2014).

[14] S. Ahlen et al., Int. J. Mod. Phys. A 25, 1 (2010).

[15] R. Massey et al., Rep. Prog. Phys. 73, 086901 (2010).

[16] S. Jung and C.S. Shin, Phys. Rev. Lett. 122, 041103 (2019).

[17] F. Courbin and D. Minniti, Publ. Astron. Soc. Pac. 112, 1617 (2000).

[18] C. Renault et al., Astron. Astrophys. 324, L69 (1997).

[19] P.D. Sackett and A. Gould, Astrophys. J. 419, 648 (1993).

[20] X.-P. Wu, Astrophys. J. 435, 66 (1994).

[21] C. Alcock et al., Astrophys. J. 491, 436 (1997).

[22] A. Gould, Astrophys. J. 606, 319 (2004).

[23] C.L. Thomas et al., Astrophys. J. 631, 906 (2005).

[24] K. Griest and W. Hu, Astrophys. J. 397, 362 (1992).

[25] D.P. Bennett et al., AIP Conf. Proc. 336, 77 (1995).

[26] S. Capozziello et al., J. Cosm. Astrop. Phys. 7, 24 (2013).

[27] S. Capozziello et al., Month. Not. Roy. Astron. Soc. 375, 1423 (2007).

[28] A.P. Naik et al., Month. Not. Roy. Astron. Soc. 480, 5211 (2018)

[29] V.F. Cardone et al., Month. Not. Roy. Astron. Soc. 406, 1821 (2010).

[30] S. Dodelson and M. Liguori, Phys. Rev. Lett. 97, 231301 (2006).

[31] V. Acquaviva et al., Phys. Rev. D 71, 104025 (2005).

[32] M.V. Bebronne and P.G. Tinyakov, Phys. Rev. D 76, 084011 (2007).

[33] K. Koyama and R. Maartens, J. Cosm. Astrop. Phys. 1, 16 (2006).

[34] S. Pal, Phys. Rev. D 74, 024005 (2006).

[35] D. Clowe et al., Astrophys. J. 648, L109 (2006).

[36] C. G. Boehmer and T. Harko, JCAP 0706 (2007) 025 arXiv:0705.4158 [astro-ph]].

[37] P. Sikivie and Q. Yang, Phys. Rev. Lett. 103, 111301 (2009).

[38] T. Harko, JCAP 1105 (2011) 022 arXiv:1105.2996 [astro-ph.CO]].

[39] P. H. Chavanis and T. Harko, Phys. Rev. D 86 (2012) 064011 arXiv:1108.3986 [astro-ph.SR]].

[40] T. Harko et al., J. Cosm. Astrop. Phys. 11, 027 (2015).

[41] D.C. Aveline et al., Nature 582, 193 (2020).

[42] M. Pospelov et al., Phys. Lett. B 662, 53 (2008).

[43] S. Chang et al., Phys. Rev. D 89, 015011 (2014).

[44] M.L. Graesser et al., J. High Ener. Phys. 2011, 110 (2011).

[45] L. Roszkowski, Nucl. Phys. B Proc. Suppl. 124, 30 (2003).

[46] C. Liu and J.-S. Lu, J. High Ener. Phys. 2013, 40 (2013).

[47] G. Jungman et al., Phys. Rep. 267, 195 (1996).

[48] D. Spolyar et al., Astrophys. J. 705, 1031 (2009).

[49] K. Freese et al., Rep. Prog. Phys. 79, 066902 (2016).
[50] J. Casanellas and I. Lopes, Month. Not. Roy. Astron. Soc. 410, 535 (2011).

[51] I. Lopes et al., Phys. Rev. D 83, id. 063521 (2014).

[52] P. Scott et al., Month. Not. Roy. Astron. Soc. 394, 82 (2009).

[53] J. Lopes and J. Silk, The Astrophysical Journal, 786, id. 25 (2014).

[54] J. Lopes et al., The Astrophysical Journal Letters, 880, L25 (2019).

[55] J. Lopes and I. Lopes The Astrophysical Journal, 879, id. 50 (2019).

[56] M.A. Perez-Garcia et al., Phys. Rev. Lett. 105, 141101 (2010).

[57] J.M. Lattimer and M. Prakash, Science 304, 536 (2004).

[58] J.M. Lattimer and M. Prakash, Astrophys. J. 550, 426 (2001).

[59] C. Alcock et al., Astrophys. J. 310, 261 (1986).

[60] C. Kouvaris and P. Tinyakov, Phys. Rev. D 82, 063531 (2010).

[61] Y. Kurita and H. Nakano, Phys. Rev. D 93, 023508 (2016).

[62] E.J.M. Madarassy and V.T. Toth, Phys. Rev. D 91, 044041 (2015).

[63] X.Y. Li et al., J. Cosm. Astrop. Phys. 06, 001 (2012).

[64] X. Li, F. Wang and K. S. Cheng, JCAP 1210 (2012) 031 arXiv:1210.1748 [astro-ph.CO]].

[65] G. Panotopoulos and I. Lopes, Phys. Rev. D 96 (2017) no.2, 023002 arXiv:1706.07272 [gr-qc]].

[66] G. Panotopoulos and I. Lopes, Phys. Rev. D 96 (2017) no.8, 083013 arXiv:1709.06643 [gr-qc]].

[67] G. Panotopoulos and I. Lopes, Int. J. Mod. Phys. D 27 (2018) no.09, 1850093 doi:10.1142/S0218271818500931 arXiv:1804.05023 [gr-qc]].

[68] I. Lopes and G. Panotopoulos, Phys. Rev. D 97, 024030 (2018).

[69] G. Panotopoulos and I. Lopes, Phys. Rev. D 97, 024025 (2018).

[70] A. A. Starobinsky, Phys. Lett. B 91, 99 (1980).

[71] P. Jetzer, Phys. Rep. 220, 163 (1992).

[72] F.E. Schunck and E.W. Mielke, Class. Quant. Grav. 20, R301 (2003).

[73] M. Gleiser, Phys. Rev. D 38, 2376 (1988).

[74] A.R. Liddle and M.S. Madsen, Int. J. Mod. Phys. D 1, 101 (1992).

[75] M. Colpi et al., Phys. Rev. Lett. 57, 2485 (1986).

[76] J.F.M. Delgado et al., J. Cosm. Astrop. Phys. 06, 037 (2020).

[77] H.-B. Li et al., Phys. Rev. D 101, 044017 (2020).

[78] M. Choptuik et al., Phys. Rev. Lett. 123, 131101 (2019).

[79] D. Guerra et al., J. Cosm. Astrop. Phys. 09, 061 (2019).

[80] A. Maselli et al., Phys. Rev. D 96, 023005 (2017).

[81] H. Olivares et al., Month. Not. Roy. Astron. Soc. 497, 521 (2020).

[82] D.F. Torres et al., Phys. Rev. D 62, 104012 (2000).

[83] M.P. Dabrowski and F.E. Schunck, Astrophys. J. 535, 316 (2000).

[84] F.H. Vincent et al., Class. Quant. Grav. 33, 105015 (2016).

[85] J. Bramante et al., Phys. Rev. D 87, 055012 (2013).

[86] C.F.B. Macedo et al., Phys. Rev. D 88, 064046 (2013).

[87] M.K. Mak and T. Harko, Roy. Soc. Lon. Proc. Ser. A 459, 393 (2003).

[88] K. Dev and M. Gleiser, Gen. Rel. Grav. 35, 1435 (2003).

[89] G. Raposo et al., Phys. Rev. D 99, 104072 (2019). 
[90] A.A. Isayev, Phys. Rev. D 96, 083007 (2017).

[91] H. Heintzmann and W. Hillebrandt, Astron. Astrophys. 38, 51 (1975).

[92] W. Hillebrandt and K.O. Steinmetz, Astron. Astrophys. 53, 283 (1976).

[93] H.O. Silva et al., Class. Quant. Grav. 32, 145008 (2015).

[94] V. Folomeev, Phys. Rev. D 97, 124009 (2018).

[95] D.D. Doneva and S.S. Yazadjiev, Phys. Rev. D 85, 124023 (2012).

[96] V.A. Torres-Sánchez and E. Contreras, Eur. Phys. J. C 79, 829 (2019).

[97] A.M. Setiawan and A. Sulaksono, AIP Conf. Proc. 1862, 030001 (2017).

[98] A.M. Setiawan and A. Sulaksono, Eur. Phys. J. C 79, 755 (2019).

[99] R. Sharma and S. D. Maharaj, Mon. Not. Roy. Astron. Soc. 375 (2007), 1265-1268 arXiv:gr-qc/0702046 [grqc]].

[100] I. Lopes, G. Panotopoulos and Á. Rincón, Eur. Phys. J. Plus 134, no. 9, 454 (2019) arXiv:1907.03549 [gr-qc]].

[101] R. C. Tolman, Phys. Rev. 55, 364 (1939).

[102] J. R. Oppenheimer and G. M. Volkoff, Phys. Rev. 55 (1939) 374.

[103] R. Brito, V. Cardoso, C. A. R. Herdeiro and E. Radu, Phys. Lett. B 752 (2016), 291-295 doi:10.1016/j.physletb.2015.11.051 arXiv:1508.05395 [gr-qc]].

[104] C. A. R. Herdeiro, G. Panotopoulos and E. Radu, JCAP 2008 (2020) 029 doi:10.1088/1475-7516/2020/08/029 arXiv:2006.11083 [gr-qc]].

[105] Kaup, D. J., Phys. Rev. 172 (1968) 1331.
[106] Ruffini, R. and Bonazzola, S., Phys. Rev. 187 (1969) 1767.

[107] Kusmartsev, F. V., Mielke, E. W. and Schunck, F. E., Phys. Rev. D 43 (1991) 3895.

[108] S. L. Liebling and C. Palenzuela, Living Rev. Rel. 20 (2017) no.1, 5 doi:10.12942/lrr-2012-6 arXiv:1202.5809 [gr-qc]].

[109] C. F. B. Macedo, P. Pani, V. Cardoso and L. C. B. Crispino, Phys. Rev. D 88 (2013) no.6, 064046 doi:10.1103/PhysRevD.88.064046 arXiv:1307.4812 [grqc]].

[110] V. Cardoso, E. Franzin, A. Maselli, P. Pani and G. Raposo, Phys. Rev. D 95 (2017) no.8, 084014 Addendum: [Phys. Rev. D 95 (2017) no.8, 089901] doi:10.1103/PhysRevD.95.089901, RevD.95.084014 arXiv:1701.01116 [gr-qc]].

[111] H. Bondi, Proc. R. Soc. Lond. A 281 (1964) 39.

[112] M. K. Mak and T. Harko, Chin. J. Astron. Astrophys. 2, 248 (2002).

[113] D. Deb, S. Roy Chowdhury, S. Ray and F. Rahaman, Gen. Rel. Grav. 50, no. 9, 112 (2018) arXiv:1509.00401 [gr-qc]].

[114] D. Deb, S. R. Chowdhury, S. Ray, F. Rahaman and B. K. Guha, Annals Phys. 387, 239 (2017) arXiv:1606.00713 [gr-qc]].

[115] P. Bhar, M. Govender and R. Sharma, Eur. Phys. J. C 77, no. 2, 109 (2017) arXiv:1607.06664 [gr-qc]].

[116] G. Panotopoulos and A. Rincón, Eur. Phys. J. Plus 134, no. 9, 472 (2019) arXiv:1907.03545 [gr-qc]]. 